\title{
Regularization of PET Reconstruction Using Multi-scale Adaptive Thresholding
}

\author{
Yinpeng Jin $^{1}$, Peter Esser ${ }^{2}$, Taro Aikawa ${ }^{2}$, Barbara Kuang ${ }^{2}$, Sheila Duan ${ }^{2}$, Andrew Laine ${ }^{1,2}$ \\ ${ }^{1}$ Department of Biomedical Engineering, Columbia University, NY, USA \\ ${ }^{2}$ Department of Radiology, Columbia University, NY, USA
}

\begin{abstract}
A multi-scale adaptive thresholding scheme is presented in this study. It was evaluated as a regularization process to filtered back-projection (FBP) for reconstructing clinical PET brain data. Adaptive selection of thresholding operators for each multi-scale sub-band enabled a unified process for noise removal and feature enhancement. A crossscale regularization process was utilized as an effective signal recovering operator. Together with non-linear thresholding and enhancement operators, they offered remarkable postprocessing to FBP reconstructed data. In addition, such effectiveness was formulated as a regularization process to optimize FBP reconstruction. A comparison study with multiscale regularized FBP (MFBP), standard FBP with clinical settings and iterative reconstruction (OSEM) was reported. The proposed regularization process has shown competitive improvement in the image quality of $P E T$ reconstructions when compared to the current state-of-the-art method used in clinical commercial systems (OSEM).
\end{abstract}

Keywords-PET, multi-scale de-noising, dyadic wavelets, cross-scale regularization, filtered back-projection.

\section{INTRODUCTION}

In clinical systems, the most often used tomographic reconstruction method is based on a filtered back-projection (FBP) algorithm [1]. Mathematically, a 2D inverse radon transform can be implemented by first applying a ramp filter to the input sinogram and then "back-projecting" the filtered data into a planar image. A ramp filter is a typical high-pass filter, which amplifies high frequency components of the input data. When noise exists, it usually occupies higher frequency sections of the spectrum. Using a ramp filter in the FBP process therefore causes the problem of noise amplification. Because of the noise and statistical fluctuations associated with nuclear decay, compounded by acquisition constraints, such as suboptimal sampling and the effects of attenuation, scatter, and collimator and detector constraints, high levels of noise almost always exists in clinical PET data. A regularization filtering process is often needed in tomographic reconstruction to alleviate the noise amplification problem.

It is very common to combine a low pass filter together with the ramp filter to eliminate part of the high frequency spectrum. Using a low-pass filter suppresses the high frequency noise, but at a sacrifice of image contrast and resolution, as well as detailed spatial information. One of the most important procedures in tomographic reconstruction is to find a best trade-off between signal-to-noise ratio and contrast/resolution of the reconstructed image. In clinical environments, post-processing involving de-noising and enhancement is often applied to improve image quality of the reconstructed data.

Wavelets have been applied to tomographic imaging in many aspects. The most popular application is local reconstruction to improve spatial resolution within a region of interest [2-6]. With the advantage of multi-resolution analysis, wavelets have also been used to accelerate implementations of the traditional FBP algorithm [7, 8].

As a successful de-noising tool, wavelet methods of analysis were also used as post-filtering or regularization/constraints to tomographic reconstruction [912].

An effective de-noising technique to tomgraphic images (PET and SPECT) can consist of:

1. Post-processing of tomographic (PET/SPECT) images reconstructed using clinical protocol.

2. Regularization of FBP to improve the reconstruction image quality.

\section{MethodOLOGY}

\section{A. Wavelet Modulus Analysis}

Using the first derivative of a cubic spine function as the wavelet bases, the three components of a 3-D dyadic wavelet coefficient $W_{m}^{k} s\left(n_{1}, n_{2}, n_{3}\right)=<s, \psi_{m, n_{1}, n_{2}, n_{3}}^{k}>, k=1,2,3$ are proportional to the coordinate components of the gradient vector of an input image $s$ smoothed by a dilated version of a cubic spine function $\theta$. From these coordinate components, one can compute the direction of the gradient vector, which indicates the direction in which the first derivative of the smoothed $s$ has the largest amplitude (or the direction in which $s$ changes the most rapidly in a local neighborhood). The amplitude of this maximized first derivative is equal to the modulus of the gradient vector, and therefore proportional to the wavelet modulus [12, 13]:

$$
M_{m} f=\sqrt{\left|W_{m}^{1} f\right|^{2}+\left|W_{m}^{2} f\right|^{2}+\left|W_{m}^{3} f\right|^{2}} .
$$

Applying a threshold value to the wavelet modulus, is equivalent to selecting first a direction in which the partial derivative is maximum at each scale, and thresholding the amplitude of the partial derivatives in this direction. The coefficients of the dyadic wavelet expansion are then computed from the thresholded modulus and the direction of the gradient vector (which was preserved during the thresholding process). Such a paradigm applies an 
adaptive choice of the spatial orientation in order to best correlate the signal. It therefore provides a more flexible and accurate orientation analysis to correlated signals when compared to traditional thresholding schemes that analyze on three orthogonal Cartesian directions separately. The flexibility and accuracy of these orientation analyses is especially beneficial in higher dimensional space.

\section{B. Multi-scale Adaptive Thresholding}

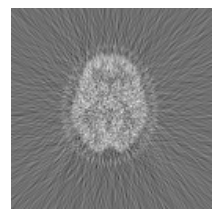

(a)

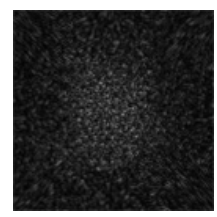

(b)

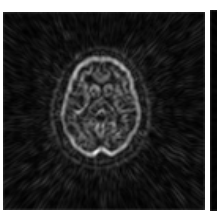

(c)

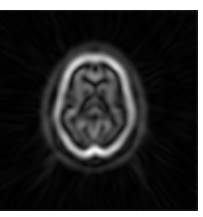

(d)

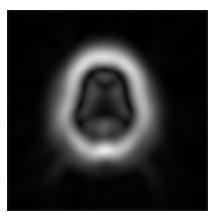

(e)
Figure 1: (a) Sample PET image reconstructed using FBP with Ramp filter. (b)-(e): 3-D wavelet modulus at expansion level 1-4.

An example of 3-D wavelet modulus representation computed from a PET brain image is shown in Figure 1. Although noise is noticeable in dyadic expansion levels 2-4, they appeared to have significantly smaller magnitude when compared to signal variations, and therefore can be easily removed by applying a simple threshold operator. On the other hand, wavelet coefficients in the most detailed expansion level show overwhelming amounts of noise. This observation suggested that substantially different signal-tonoise relations exist within distinct sub-bands of wavelet coefficients. With such considerations, we adaptively select suitable thresholding and enhancement operators based on the signal-noise characteristics for each expansion sub-band. More specifically, for clinical PET brain data used in this study the follow is noted:

1. For the first expansion level, as shown in Figure 1 (b), traditional thresholding operator will not be able to recover signal related feature. Therefore a more sophisticated "thresholding" scheme was applied (e.g. cross-scale regularization [14]).

2. The second expansion level, as shown in Figure 1 (c), contained detailed structural information. A piece-wise linear enhancement operator [15] was applied to increase the strength of signal features.

3. Higher levels of wavelet sub-bands were processed using an affine threshold operator [16] for de-noising.

\section{Cross-scale Regularization}

To recover signal related features in noise dominated wavelet sub-bands, a cross-scale regularization was suggested [14]. First, an edge indication map was constructed using the next higher level of wavelet subbands. Then, a selected wavelet sub-band was multiplied with the edge map to preserve signal related wavelet coefficients.

The success of this cross-scale regularization process came from the truth that random noise tends to have a different singularity (negative Lipschitz regularity) from coherent signal features, and therefore decreases steeply when wavelet scales increase [17]. Thus, noise components usually have a very low coherence across wavelet expansion levels.

For images with high levels of noise, the cross-scale regularization offers superior capability of recovering detailed signal features when compared to conventional thresholding schemes. An example is shown in Figure 2.

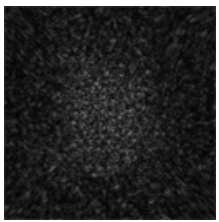

(a)

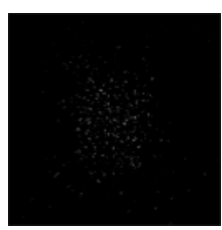

(b)

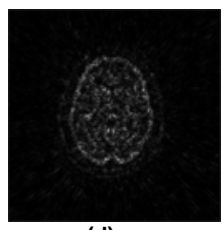

(d)

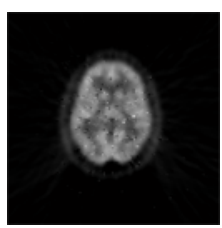

(c)

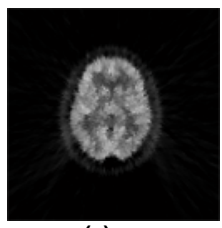

(e)
Figure 2: Comparison of wavelet shrinkage [18] and cross-scale regularization. (a) pre-processed wavelet modulus at expansion level 1, as shown in Figure 1 (b). Thresholded wavelet modulus by (b) soft threshold, (d) cross-scale regularization. And the final denoising result of (c) wavelet shrinkage, and (e) cross-scale regularization.

\section{Multi-scale Regularized FBP (MFBP)}

By embedding a multi-scale de-noising module as an extra regularization process as an alternative to the traditional low-pass filter, we can expect an improved tomographic reconstruction. The simplest way to implement such an idea is to include more high frequency features during the FBP reconstruction (using a low-pass filter with a limited high frequency cut-off parameter). The strategy is that additional amount of noise accompanied with detailed information of the signal can be recovered by more sophisticated de-noising.

In this study, MFBP images were generated as follows:

1. Raw data was reconstructed using FBP with a standard ramp filter on a clinical workstation.

2. Multi-scale adaptive thresholding was applied to the raw data for de-noising and enhancement. 


\section{EXPERIMENTAL DESIGN}

Two radiologists specialized in nuclear radiology and nuclear medicine participated in the comparison study. They were asked to review 30 comparative trials. Each trial contained three different reconstructions (FBP with clinical setting, OSEM with clinical setting, and multi-scale regularized FBP) of the same clinically acquired PET brain data. The three reconstructions were displayed in random order. Without explicit knowledge about the reconstruction process of each compared data, the readers were asked to give subjective scores (with a number between 1 and 10, 1 indicating "poor" image quality, and 10 indicates the "outstanding" image quality). The following characteristics reflecting image quality of PET data were recorded:

1. Noise. A subjective evaluation for signal-to-noise ratio, which reflects the effectiveness of multi-scale regularization.

2. Contrast. Radiologists often comment on image data's "sharpness", which reflects the visibility of major anatomical and physiological structures.

3. Details. We asked radiologist to point out whether or not more detailed structures could be observed, which reflects the spatial resolution of the reconstructions.

4. Overall Image Quality. In addition to the above scorings, the radiologists were also asked to give a score on based on their overall clinical preference to the three candidate images.

A preferred color-map was used by each radiologist for the evaluation readings, as shown in Figure 3.

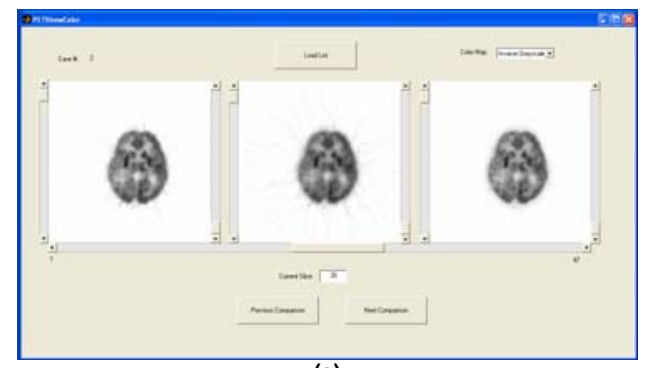

(a)

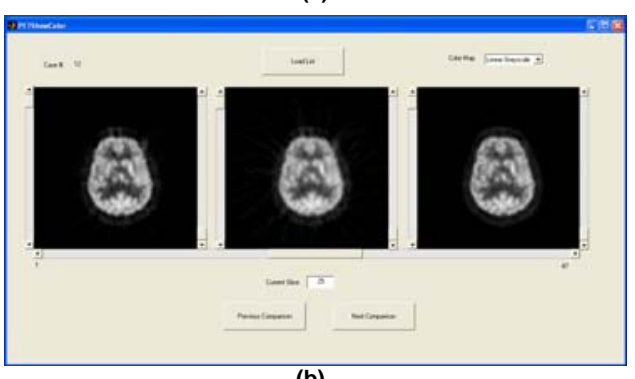

(b)

Figure 3: The graphical user interface (GUI) used in the comparison study. (a) Comparison using inverse grayscale color-map. (b) Comparison using standard linear grayscale color-map.

\section{Results}

To exclude the effect of different image quality between datasets, the evaluation scores were normalized case-by-case such that the scores for FBP data were set to 5.0 for all four factors. For OSEM and MFBP data, the scores relative to the FBP data were collected for statistical analysis.

Table 1 shows a summary of the average scores for each reader. A one-way analysis of variance (ANOVA) was used for statistical analysis of each factor. Statistical significance was claimed when $p<0.05$.

Table 1: Summary of the evaluation scores for each reader.

\begin{tabular}{|c|c|c|c|c|}
\hline (Reader 1) & Noise & Contrast & Details & Overall \\
\hline FBP & 5 & 5 & 5 & 5 \\
\hline OSEM & 4.67 & 6.47 & 6.13 & 6.60 \\
\hline MFBP & 4.87 & 7.13 & 6.93 & 6.87 \\
\hline Significance & No & Yes & Yes & $\begin{array}{c}\text { FBP to } \\
\text { (OSEM, MFBP) }\end{array}$ \\
\hline
\end{tabular}

\begin{tabular}{|c|c|c|c|c|}
\hline (Reader 2) & Noise & Contrast & Details & Overall \\
\hline FBP & 5 & 5 & 5 & 5 \\
\hline OSEM & 6.20 & 5.20 & 5.20 & 5.20 \\
\hline MFBP & 5.00 & 4.36 & 4.36 & 4.36 \\
\hline Significance & No & No & No & No \\
\hline
\end{tabular}

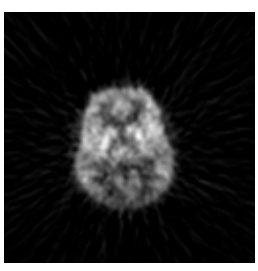

(a1)

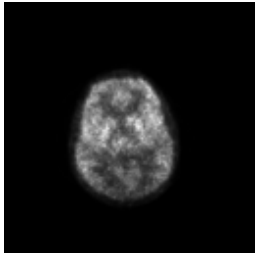

(b1)

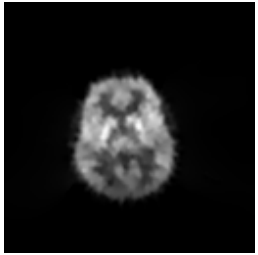

(c1)

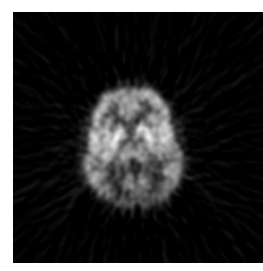

(a2)

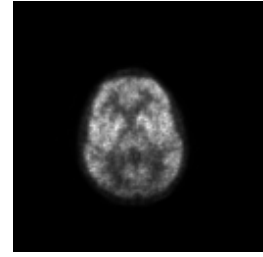

(b2)

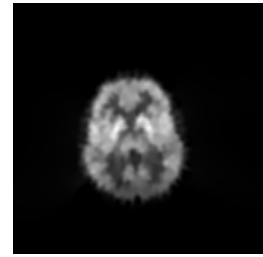

(c2)

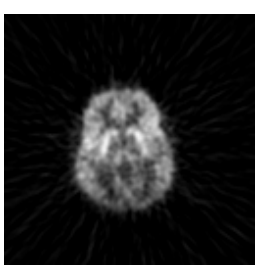

(a3)

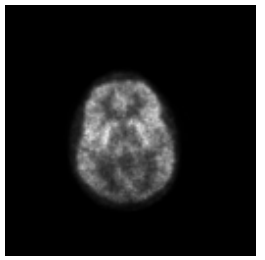

(b3)

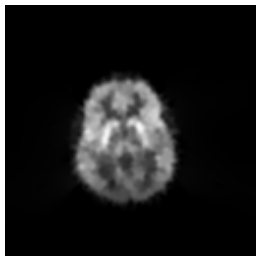

(c3)
Figure 4: Sample image data from (a) FBP, (b) OSEM, and (c) MFBP. Three contiguous slices of data are shown. 
Reader 1 noticed slightly higher levels of noise in OSEM and MFBP (without significance). MFBP data showed significant improvement in contrast and detail when compared to FBP and OSEM. The reader also showed a significant overall preference to OSEM and MFBP data when compared to FBP, while no significance was observed for the preference between OSEM and MFBP.

Reader 2 showed a stronger preference to FBP data as it was more familiar in routine clinical readings. For $52 \%$ of the cases, FBP data was considered the most preferred. However, all statistical differences were not significant. In addition, reader 2 gave the same score for contrast, details and overall image quality for all cases.

Both readers commented that MFBP and OSEM images were similar in terms of noise level, contrast and visibility of detail structures. A sample comparison is shown in Figure 4.

\section{CONCLUSION AND DISCUSSION}

We have presented in this study a general scheme of multi-scale adaptive thresholding. It explored the difference between spatial-frequency sub-bands, and adaptively applying suitable threshold operators to achieve optimal denoising and enhancement results. A cross-scale regularization process was utilized to recover subtle signal features from the finer levels of a wavelet expansion.

The effectiveness of the proposed multi-scale de-noising technique was further investigated as a regularization process to tomographic image reconstruction using FBP algorithm.

A clinical comparison study was reported to illustrate the usefulness of an advanced de-noising technique in FBP reconstructions. Preliminary result suggest that with multiscale de-noising, image quality from FBP reconstruction can be improved. The improvement can be clearly appreciated when compared to current state-of-the-art iterative methods for tomographic reconstruction, such as OSEM.

Although the radiologists participated in this study showed different levels of clinical preference to the proposed technique, statistical analysis demonstrated that multi-scale regularized FBP generated image data with superior contrast and detail.

Recent research on EM based reconstruction methods suggested that filtering and de-noising techniques are useful to improve these iterative methods. Therefore, the multiscale de-noising technique reported in this study could be used within these methods to improve reconstruction image quality.

\section{ACKNOWLEDGMENT}

This study is partially supported by research grant from Siemens Medical Solutions, USA, Inc.

\section{REFERENCES}

[1] G. Herman, Image Reconstruction from Projections: The Fundamentals of Computerized Tomography. New York NY: Academic Press, 1980.

[2] T. Olson and J. De Stefano, "Wavelet Localization of the Radon Transform." IEEE Trans. Image Processing, vol. 42, pp. 20552067, 1994.

[3] F. Rashid-Farrokhi, K. Liu, C. Berenstein, and D. Walnut, "Wavelet-based Multiresolution Local Tomography." IEEE Trans. Image Processing, vol. 22, pp. 1412-1430, 1997.

[4] S. Zhao, W. G., and J. Hsieh, "Wavelet Sampling and Localization Schemes for the Radon Transform in Two Dimensions." SIAM Journal on Applied Mathematics, vol. 57, pp. 1749-1762, 1997.

[5] M. Bottema, B. Morean, and S. Suorova, "An Application of Wavelets in Tomography." Digital Signal Processing, vol. 8, pp. 244-254, 1998.

[6] W. Maldych, "Tomography, Approximate Reconstructions, and Continuous Wavelet Transforms." Journal of Applied Computation and Harmonic Analysis, vol. 7, pp. 54-100, 1999.

[7] A. Delaney and Y. Bresler, "Multi-resolution Tomographic Reconstruction Using Wavelets." IEEE Trans. Image Processing, vol. 4, pp. 799-813, 1995.

[8] L. Blanc-Feraud, P. Charbonnier, P. Lobel, and M. Barlaud, "A Fast Tomographic Reconstruction Algorithm in the 2-D Wavelet Transform Domain." IEEE International Conference on Acoustics, Speech and Signal Processing, pp. 305-308, 1994.

[9] E. Kolaczyk, "A Wavelet Shrinkage Approach to Tomographic Image Reconstruction." Journal of American Statistics Association, vol. 91, pp. 1079-1090, 1996.

[10] N. Lee and B. Lucier, "Wavelet Methods for Inverting the Radon Transform with Noisy Data." IEEE Trans. Image Processing, vol. 10 (1), pp. 79-94, 2001.

[11] J. Lin, A. Laine, and S. Bergmann, "Improving PET-based Methods Using the Wavelet Transform for Positron Emission Tomography." IEEE Trans. Biomedical Engineering, vol. 48, pp. 202-212, 2001.

[12] J. Kalifa, A. Laine, and P. Esser, "Regularization in Tomographic Reconstruction Using Thresholding Estimators." IEEE Trans. Medical Imaging, vol. 22 (3), pp. 351-359, 2003.

[13] S. Mallat, A Wavelet Tour of Signal Processing.: Academic Press, 1998.

[14] Y. Jin, E. Angelini, P. Esser, and A. Laine, "De-noising SPECT/PET Images Using Cross-scale Regularization." Proceedings of the Sixth International Conference on Medical Image Computing and Computer Assisted Interventions (MICCAI 2003), Montreal Canada,vol. 2879 (2), pp. 32-40, 2003.

[15] A. Laine, J. Fan, and S. Schuler, "A Framework for Contrast Enhancement by Dyadic Wavelet Analysis." in Digital Mammography, A. Gale, S. Astley, D. Dance, and A. Cairns, Eds. Amsterdam, The Netherlands: Elsevier, 1994.

[16] H. Gao and A. Bruce, "WaveShrink with Firm Shrinkage." Statist. Sinica, vol. 7, pp. 855-874, 1997.

[17] S. Mallat and S. Zhong, "Characterization of Signals from Multiscale Edges." IEEE Trans. Pattern Analysis and Machine Intelligence, vol. 14, pp. 710-732, 1992.

[18] D. Donoho, "De-noising by Soft-thresholding." IEEE Trans. Information Theory, vol. 41 (3), pp. 613-627, 1995. 